\title{
Mechanism Analysis of Dynamic Compaction based on Large Deformation
}

\author{
Xie Nenggang*, Chen Yun, Ye Ye and Wang Lu
}

Anhui University of Technology, Maanshan, Anhui Province, China, 243002

\begin{abstract}
In the mechanism analysis of foundation consolidation by using dynamic compaction, many kinds of non-linear conditions exist. This paper adopts large deformation on the relationship between strain and displacement. Non-linear governing equation of soil, based on finite element method, is established. Iterative calculation form is raised. Finally, non-linear numerical analysis is done to a calculation example. We not only obtain the changing regularity of soil displacement and stress during the acting time of dynamic compaction, but also achieve their contour maps after compaction.
\end{abstract}

Keywords: Dynamic compaction, non-linear, large deformation.

\section{INTRODUCTION}

As for multiple factors and complex of the dynamic compaction(refer with Fig. 1), many scholars have studied reinforcement mechanisms of compaction from the engineering practice, laboratory tests, numerical analysis and other fields. When the basic control equations of dynamic problems are established, there are the following major principle issues: 1) Stress - strain model; 2) Strain displacement model; 3) The subjects were considered only soil skeleton, or considering using the coupled analysis of soil skeleton and pore water (Dynamic consolidation model is adopted); 4) Calculation methods of shock loads or dynamic contact stress between ground surface and the bottom hammer when tamping. The main difference existence in various studies of theory and methods of compaction problems lies in the handling of some above principle issues. In the relations of stress-strain, dynamic loading and unloading bilinear constitutive model presented by Qian Jiahuan [1] was widely used. In the straindisplacement relations, most researchers also use the "small strain" assumption. Jiang Peng [2] in proposed a "large deformation" assumption that is, considering geometric nonlinearity. Dong-Soo Kim [3] proposed that for different vibration source, the geometric models to be built were also different. In [4], only the soil skeleton was considered to do the compaction. In considering the coupling analysis of soil skeleton and pore water, Yang Jun [5] provided the time solutions on symmetric problems of fluid dynamic coupling shaft and the improved method of the problem was given by Kong Lingwei [6]. Using three-dimensional consolidation theory of porous media, Chen Jie [7] et al. calculated and analyzed the structure of saturated soil compaction process. In the numerical simulation processes of dynamic consolidation of saturated soft clay foundation, Ding Zhenzhou [8]

*Address correspondence to this author at the School of Mechanical Engineering, Anhui University of Technology, Maanshan 243002, China; Tel: +86-0555-2311041(office); E-mail: xienenggang @yahoo.com.cn

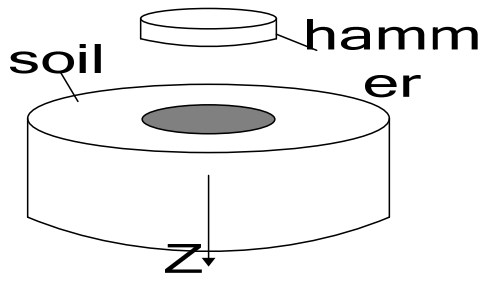

Fig. (1). Dynamic compaction action.

used Boit consolidation theory. He considered the strong nonlinear coupling problem of the soil and pore water and obtained the dynamic changes of the process of soil consolidation. Song Xiuguang [9] took fluid dynamic coupling and contact coupling between hammer and foundation soil into account and obtained changes of soil displacement and contact dynamic stress under time period in the dynamic compaction. Gunaratne $M$ [10] used the numerical method to simulate dynamic consolidation according to the modified classical Terzaghi static consolidation theory. He took the contact dynamic stress as the pulse stress which was broken down into a sufficient number of load steps so as to calculate the characteristics of pore pressure. Zhou XL [11] used Biot's consolidation theory to study the transient dynamic response of foundation under the triangular pulse load and Laplace transform to solve the problem. Then he obtained the time history between displacement and pore pressure and the relationships of load forms. Besides, he [12] also studied the dynamic response of the saturated soil under the concentrated load and got more accurate displacement, stress and the numerical solution of pore pressure. In terms of the transient load simulation generated by the dynamic compaction, it usually would be simplified to a triangle wave or half-sine based on experience [13]. The calculation of dynamic contact between the bottom of hammer and the face of soil is the key technique problem of dynamic compaction. In this field, Scott RA and Pearce RW [14] put forward a calculation forum, but it couldn't satisfy the initial condition. Kong LW and Yuan JX [15] used integral transform and transition 
matrix to study the dynamic contact stress. The technique also has been studied analytically by Roesset JM [16]. However, the method above, even many other essays, were merely in space field, explained the distributing feature of dynamic contact stress on the bottom of hammer, they adopted a simple elastic contact model in time field during the dynamic compaction, and didn't take the possible behavior of hammer into account such as, bouncing contact and separating. Jiang P and Li RQ [17] considered this nonlinear contact and suggested to adopt impulse-dynamic contact model to calculate the dynamic contact stress, but the contact condition in this model is static form.

\section{DIFFERENTIAL GOVERNING EQUATION}

For dynamic compaction, let active areas of reinforcement foundation be abstracted to be $\Omega$ which is bounded by boundary $S$, where $X$ is one of the points. Then the basic equations of the large deformation problem of soil body are as follows

Dynamic balance equation is

$\left[\left(\delta_{i k}+u_{i, k}\right) \sigma_{k j}\right]_{, j}+F_{i}-p \ddot{u}_{i}=0$

Relationship between strain and displacement is

$\varepsilon_{i j}=\frac{1}{2}\left(u_{i, j}+u_{j, i}+u_{k, i} u_{k, j}\right)$

Relationship between stress and strain is

$\sigma_{i j}=\lambda \varepsilon_{k k} \delta_{i j}+2 G \varepsilon_{i j}$

Boundary conditions of displacement $\left(S_{u}\right)$

$u_{i}=\bar{u}_{i}$

Boundary condition of stress $\left(S_{p}\right)$

$\left(\delta_{i k}+u_{i, k}\right) \sigma_{k j} n_{j}=\bar{p}_{i}$

Where : Each physical quantity is expressed as a tensor component form. That is, $u_{i}$ is a displacement component, $\sigma_{i j}$ a stress tensor component, $\varepsilon_{i j}$ a strain tensor component, $F_{i}$ a force component of the volume of soil, $\rho$ the density of soil body. Besides $\bar{u}_{i}$ and $\bar{p}_{i}$ are given boundary displacement and external borders, $\lambda$ and $G$ Lame constants, $\delta_{i j}$ a Clinton Sign and $n_{i}$ denotes direction cosine.

Energy functional is constructed as follows

$\Pi=\int_{t_{1}}^{t_{2}}\left\{\iiint_{\tau}\left[\frac{1}{2} \rho \dot{u}_{i} \dot{u}_{i}-A\left(\varepsilon_{i j}\right)+F_{i} u_{i}\right] d \tau+\iint_{S_{p}} \bar{p}_{i} u_{i} d S\right\} d t$

\section{Hamilton's Principle}

Boundary conditions of displacement and relationship between strain and displacement are met so that functional displacement $u_{i}$ of the extreme value must satisfy the dynamic equilibrium equation, stress boundary conditions and stress-strain relations under the given conditions of displacement $u_{i}$ at time $t=t_{1}$ and $t=t_{2}$, that is the true solution of the problem. Let the energy functional in equation (6) be varied into zero

$\delta \Pi=0$

\section{GOVERNING EQUATIONS BASED ON FINITE ELEMENT}

With the finite element method and the discrete structure, the energy functional for body of any unit is

$$
\begin{aligned}
& \Pi_{e}=\int_{t_{1}}^{t_{2}}\left\{\iiint_{v_{e}}\left[\frac{1}{2} \rho\{\dot{u}\}_{e}^{T}[N]^{T}[N]\{\dot{u}\}_{e}-A\left(\varepsilon_{i j}\right)\right] d v_{e}\right. \\
& \left.+\{u\}_{e}^{T}\{F\}_{e}+\{u\}_{e}^{T}\{P\}_{e}\right\} d t
\end{aligned}
$$

Where, $\{u\}_{e},\{\dot{u}\}_{e}$ denote the displacement array and velocity array of element node respectively; $[N]$ denotes the shape function matrix; $\{F\}_{e}$ is the equivalent nodal load caused by body force of the element; $\{P\}_{e}$ is the equivalent nodal load caused by surface force.

Let equation (8) be varied into

$$
\delta \Pi_{e}=\int_{t_{1}}^{t_{2}}\left\{\delta\{u\}_{e}^{T}\left(-[m]_{e}\{\ddot{u}\}_{e}+\{F\}_{e}+\{P\}_{e}\right)-\iiint_{v_{e}} \delta\{\varepsilon\}^{T}\{\sigma\} d v_{e}\right\} d t
$$

With $\{\varepsilon\}=[\bar{B}]_{e}\{u\}_{e}\left([\bar{B}]_{e}\right.$ is the strain matrix for the unit), equation (9) is transformed into

$$
\delta \Pi_{e}=\int_{t_{1}}^{t_{2}} \delta\{u\}_{e}^{T}\left\{-[m]_{e}\{\ddot{u}\}_{e}+\{F\}_{e}+\{P\}_{e}-\iiint_{v_{e}}[\bar{B}]_{e}^{T}\{\sigma\} d v_{e}\right\} d t
$$

Where, $[m]_{e}=\iiint_{v_{e}} \rho[N]^{T}[N] d v_{e}$ is the mass matrix for the unit.

Let equation (8) be varied into zero and obtain dynamic equilibrium equation of the unit body

$$
[m]_{e}\{\ddot{u}\}_{e}+\iiint_{v_{e}}[\bar{B}]_{e}^{T}\{\sigma\} d v_{e}=\{F\}_{e}+\{P\}_{e}
$$

According to the unit assembly, the dynamic equation of overall structure can be obtained

$$
[M]\{\ddot{u}\}+\sum_{e} \iiint_{v_{e}}[\bar{B}]_{e}^{T}\{\sigma\} d v_{e}=\{F\}+\{P\}
$$

\section{METHOD FOR SOLVING THE NONLINEAR DYNAMIC EQUATIONS}

\subsection{Dynamic Equation in Incremental Form}

According to equation (2), relations of straindisplacement belong to nonlinear. So $[\bar{B}]_{e}$ is the function of $\{u\}_{e}$ and can be written as

$[\bar{B}]_{e}=\left[B_{0}\right]+\left[B_{L}\right]_{e}$

Where: $\left[B_{0}\right]$ is the matrix entry by linear deformation analysis; $\left[B_{L}\right]_{e}$ is the matrix entry by nonlinear deformation, 
which is relative to the node displacement $\{u\}_{e}$ of the unit body.

Equation (11) can be rewritten as

$$
\phi\left[\{u\}_{e}\right]=[m]_{e}\{\ddot{u}\}_{e}+\iiint_{v_{e}}[\bar{B}]_{e}^{T}\{\sigma\} d v_{e}-\left(\{F\}_{e}+\{P\}_{e}\right)=0
$$

Let $\{u\}_{e}$ be derived on both sides in equation (14) and we can obtain

$$
d \phi\left[\{u\}_{e}\right]=\iiint_{v_{e}} d[\bar{B}]_{e}^{T}\{\sigma\} d v_{e}+\iiint_{v_{e}}[\bar{B}]_{e}^{T} d\{\sigma\} d v_{e}
$$

As

$$
\{\sigma\}=[D]\left(\{\varepsilon\}-\left\{\varepsilon_{0}\right\}\right)+\left\{\sigma_{0}\right\}
$$

Where: $[D]$ is an elastic matrix; $\left\{\varepsilon_{0}\right\},\left\{\sigma_{0}\right\}$ are the initial strain and initial stress respectively.

Hence,

$d\{\sigma\}=[D] d\{\varepsilon\}=[D][\bar{B}]_{e} d\{u\}_{e}$

In equation (13), $\left[B_{0}\right]$ is irrelative to the node displacement $\{u\}_{e}$. Therefore,

$d[\bar{B}]_{e}=d\left[B_{L}\right]_{e}$

Type equations (17) and (18) into equation (15) and we can get

$d \phi\left[\{u\}_{e}\right]=\iiint_{v_{e}} d\left[B_{L}\right]_{e}^{T}\{\sigma\} d v_{e}+[\bar{k}]_{e} d\{u\}_{e}$

Where:

$[\bar{k}]_{e}=\iiint_{v_{e}}[\bar{B}]_{e}^{T}[D][\bar{B}]_{e} d v_{e}=\left[k_{0}\right]_{e}+\left[k_{L}\right]_{e}$

Type equation (13) into (20) and we can obtain

$\left[k_{0}\right]_{e}=\iiint_{v_{e}}\left[B_{0}\right]^{T}[D]\left[B_{0}\right] d v_{e}$

$\left[k_{L}\right]_{e}=\iiint_{v_{e}}\left\{\left[B_{0}\right]^{T}[D]\left[B_{L}\right]_{e}+\left[B_{L}\right]_{e}^{T}[D]\left[B_{L}\right]_{e}+\left[B_{L}\right]_{e}^{T}[D]\left[B_{0}\right]\right\} d v_{e}$

Let

$\iiint_{v_{e}} d\left[B_{L}\right]_{e}^{T}\{\sigma\} d v_{e}=\left[k_{\sigma}\right]_{e} d\{u\}_{e}$

Where: $\left[k_{\sigma}\right]_{e}$ is the geometric stiffness matrix.

Type equations from (20) to (23) into (19) and we can get

$d \phi\left[\{u\}_{e}\right]=\left\{\left[k_{0}\right]_{e}+\left[k_{L}\right]_{e}+\left[k_{\sigma}\right]_{e}\right\} d\{u\}_{e}$

Assume that the system under external loads, element node displacement at time $\mathrm{t}$ is $\{u(t)\}_{e}$. Then the first-order for (14) at $\{u(t)\}_{e}+\Delta\{u\}_{e}$ is approximately
$\phi\left[\{u(t)\}_{e}+\Delta\{u\}_{e}\right]=\phi\left[\{u(t)\}_{e}\right]$

$+\left.\left(\frac{d \phi}{d\{u\}_{e}} \Delta\{u\}_{e}\right)\right|_{\{u\}_{e}=\{u(t)\}_{e}}=0$

Type (14) and (24) into (25) and we can obtain

$\phi\left[\{u(t)\}_{e}+\Delta\{u\}_{e}\right]=[m]_{e}\{\ddot{u}(t)\}_{e}$

$+\iiint_{v_{e}}[\bar{B}]_{e}^{T}\{\sigma\} d v_{e}-\left(\{F(t)\}_{e}+\{P(t)\}_{e}\right)$

$+\left\{\left[k_{0}\right]_{e}+\left[k_{L}\right]_{e}+\left[k_{\sigma}\right]_{e}\right\} \Delta\{u\}_{e}=0$

According to (16) and we take initial strain and initial stress as zero, then we can get

$\iiint_{v_{e}}[\bar{B}]_{e}^{T}\{\sigma\} d v_{e}=\left(\left[k_{0}\right]_{e}+\left[k_{L}\right]_{e}\right)\{u(t)\}_{e}$

Type (27) into (26), and according to the unit assembly and considering damp, we can obtain the overall dynamic equilibrium equation in the incremental form.

$$
\begin{aligned}
& \phi[\{u(t)\}+\Delta\{u\}]=[M]\{\ddot{u}(t)\}+[C]\{\dot{u}(t)\} \\
& +\left[K^{\prime}\right]\{u(t)\}-(\{F(t)\}+\{P(t)\})+[K] \Delta\{u\}=0
\end{aligned}
$$

Where: $[K]=\sum_{e}\left\{\left[k_{0}\right]_{e}+\left[k_{L}\right]_{e}+\left[k_{\sigma}\right]_{e}\right\}, \quad\left[K^{\prime}\right]=\sum_{e}\left\{\left[k_{0}\right]_{e}+\left[k_{L}\right]_{e}\right\}$, $[C]$ is the damping matrix.

\subsection{Iterative Solution}

According to the time step $\Delta t$, dynamic impact time is divided into several time periods.

1) For any time $t$, let $\{u(t)\}_{\text {small }},\{\dot{u}(t)\}_{\text {small }}$ and $\{\ddot{u}(t)\}_{\text {small }}$ which are obtained by solving the small deformation dynamic equation of the system be the initial iterative values $\{u(t)\}_{1},\{\dot{u}(t)\}_{1}$ and $\{\ddot{u}(t)\}_{1}$ of large deformation dynamic equation. Small deformation dynamic equation of the system is

$[M]\{\ddot{u}\}+\left[C_{0}\right]\{\dot{u}\}+\left[K_{0}\right]\{u\}=\{F\}+\{P\}$

Where: $\left[K_{0}\right]=\sum_{e}\left[k_{0}\right]_{e} ;\left[C_{0}\right]=\alpha[M]+\beta\left[K_{0}\right]$.

2) According to the nonlinear relationships between the strain and displacement of large deformation problem, we type $\{u(t)\}_{1}$ into (29) and get $\left[B_{L}\right]_{e}$ for every element. Then on the basis of (21), (22) and (23), $\left[k_{0}\right]_{e}$, $\left[k_{L}\right]_{e},\left[k_{\sigma}\right]_{e}$ are obtained for every element and $\left[K^{\prime}\right]_{1}$ and $[K]_{1}$ are got through assembly.

3) Type $\{u(t)\}_{1},\{\dot{u}(t)\}_{1},\{\ddot{u}(t)\}_{1},\left[K^{\prime}\right]_{1}$ and $[K]_{1}$ into (28) and get $\Delta\{u\}_{1}$

4) Using linear acceleration method, we get the values of the next iteration $\{u(t)\}_{2},\{\dot{u}(t)\}_{2}$ and $\{\ddot{u}(t)\}_{2}$ at time $\mathrm{t}$.

5) Repeat steps from 2) to 4), the iterative format is 


$$
\left\{\begin{array}{l}
\Delta\{u\}_{n}=-[K]_{n}^{-1}\left([M]\{\ddot{u}(t)\}_{n}+[C]_{n}\{\dot{u}(t)\}_{n}+\left[K^{\prime}\right]_{n}\{u(t)\}_{n}-[\{F(t)\}+\{P(t)\}]\right) \\
\{u(t)\}_{n+1}=\{u(t)\}_{n}+\Delta\{u\}_{n} \\
\{\dot{u}(t)\}_{n+1}=\{u(t)\}_{n+1} \frac{3}{\Delta t}-\{u(t-\Delta t)\} \frac{3}{\Delta t} \\
-2\{\dot{u}(t-\Delta t)\}-\{\ddot{u}(t-\Delta t)\} \frac{\Delta t}{2} \\
\{\ddot{u}(t)\}_{n+1}=\{u(t)\}_{n+1} \frac{6}{\Delta t^{2}}-\{u(t-\Delta t)\} \frac{6}{\Delta t^{2}} \\
-\{\dot{u}(t-\Delta t)\} \frac{6}{\Delta t}-2\{\ddot{u}(t-\Delta t)\}
\end{array}\right.
$$

Where: $\Delta t$ is the time step; $\{u(t-\Delta t)\},\{\dot{u}(t-\Delta t)\}$ and $\{\ddot{u}(t-\Delta t)\}$ are, respectively, the displacement, velocity and acceleration at $t-\Delta t$ for the nonlinear problem of (28).

\section{STRAIN MATRIX OF AXISYMMETRIC PROBLEM BASED ON THE GEOMETRIC NONLINEARITY}

For axisymmetric problems, we use the cylindrical coordinates $(r, \theta, z)$. Let $z$ be the axis of symmetry and all the stress, strain and displacement have nothing to do with $\theta$ but the functions of $r$ and $z$. There are only two displacement components at any point, that is, the radial displacement along to $r$ and axial displacement along the direction of $z$. Because of the symmetry, the circumferential displacement of the direction $\theta$ is zero.

Take a unit cell and element nodes are $i, j, m, \cdots$, where the displacement array of element nodes is

$$
\{\delta\}_{e}=\left\{\begin{array}{lllllll}
u_{i} & w_{i} & u_{j} & w_{j} & u_{m} & w_{m} & \cdots
\end{array}\right\}^{T}
$$

Unit displacement of any point can be expressed as

$$
\{\delta\}=\left\{\begin{array}{l}
u \\
w
\end{array}\right\}=[N]\{\delta\}_{e}
$$

Where: $[N]=\left[\begin{array}{ccccccc}N_{i} & 0 & N_{j} & 0 & N_{m} & 0 & \cdots \\ 0 & N_{i} & 0 & N_{j} & 0 & N_{m} & \cdots\end{array}\right]$.

The nonlinear relationship between strain and displacement is

$$
\{\varepsilon\}=\left\{\begin{array}{c}
\varepsilon_{r} \\
\varepsilon_{\theta} \\
\varepsilon_{z} \\
\varepsilon_{r z}
\end{array}\right\}=\left\{\begin{array}{c}
\frac{\partial u}{\partial r} \\
\frac{u}{r} \\
\frac{\partial w}{\partial z} \\
\frac{1}{2}\left(\frac{\partial u}{\partial z}+\frac{\partial w}{\partial r}\right)
\end{array}\right\}+\frac{1}{2}\left\{\begin{array}{c}
\frac{\partial u}{\partial r} \cdot \frac{\partial u}{\partial r}+\frac{\partial w}{\partial r} \cdot \frac{\partial w}{\partial r} \\
\frac{\partial u}{\partial z} \cdot \frac{u}{r} \\
\frac{\partial u}{\partial z}+\frac{\partial u}{\partial z}+\frac{\partial w}{\partial z} \cdot \frac{\partial w}{\partial z}
\end{array}\right\}
$$

Let

$$
\begin{aligned}
& \left\{\varepsilon_{0}\right\}=\left\{\begin{array}{c}
\frac{\partial u}{\partial r} \\
\frac{u}{r} \\
\frac{\partial w}{\partial z} \\
\frac{1}{2}\left(\frac{\partial u}{\partial z}+\frac{\partial w}{\partial r}\right)
\end{array}\right\} \\
& =\left\{\begin{array}{c}
\frac{\partial N_{i}}{\partial r} u_{i}+\frac{\partial N_{j}}{\partial r} u_{j}+\frac{\partial N_{m}}{\partial r} u_{m}+\cdots \\
\frac{N_{i}}{r} u_{i}+\frac{N_{j}}{r} u_{j}+\frac{N_{m}}{r} u_{m}+\cdots \\
\frac{\partial N_{i}}{\partial z} w_{i}+\frac{\partial N_{j}}{\partial z} w_{j}+\frac{\partial N_{m}}{\partial z} w_{m}+\cdots \\
\frac{1}{2}\left[\left(\frac{\partial N_{i}}{\partial z} u_{i}+\frac{\partial N_{j}}{\partial z} u_{j}+\frac{\partial N_{m}}{\partial z} u_{m}+\cdots\right)+\left(\frac{\partial N_{i}}{\partial r} w_{i}+\frac{\partial N_{j}}{\partial r} w_{j}+\frac{\partial N_{m}}{\partial r} w_{m}+\cdots\right)\right]
\end{array}\right\}
\end{aligned}
$$$$
=\left[\begin{array}{ccccccc}
\frac{\partial N_{i}}{\partial r} & 0 & \frac{\partial N_{j}}{\partial r} & 0 & \frac{\partial N_{m}}{\partial r} & 0 & \ldots \\
\frac{N_{i}}{r} & 0 & \frac{N_{j}}{r} & 0 & \frac{N_{m}}{r} & 0 & \ldots \\
0 & \frac{\partial N_{i}}{\partial z} & 0 & \frac{\partial N_{j}}{\partial z} & 0 & \frac{\partial N_{m}}{\partial z} & \ldots \\
\frac{1}{2} \cdot \frac{\partial N_{i}}{\partial z} & \frac{1}{2} \cdot \frac{\partial N_{i}}{\partial r} & \frac{1}{2} \cdot \frac{\partial N_{j}}{\partial z} & \frac{1}{2} \cdot \frac{\partial N_{j}}{\partial r} & \frac{1}{2} \cdot \frac{\partial N_{m}}{\partial z} & \frac{1}{2} \cdot \frac{\partial N_{m}}{\partial r} & \ldots
\end{array}\right]\left[\begin{array}{c}
u_{i} \\
w_{i} \\
u_{j} \\
w_{j} \\
u_{m} \\
w_{m} \\
\vdots
\end{array}\right\}
$$$$
=[L][N]\{\delta\}_{e}
$$

Where: $[L]=\left[\begin{array}{cc}\frac{\partial}{\partial r} & 0 \\ \frac{1}{r} & 0 \\ 0 & \frac{\partial}{\partial z} \\ \frac{1}{2} \cdot \frac{\partial}{\partial z} & \frac{1}{2} \cdot \frac{\partial}{\partial r}\end{array}\right]$.

Therefore, the strain matrix of small displacement is

$$
\left[B_{0}\right]=[L][N]
$$

$$
\left\{\varepsilon_{L}\right\}=\frac{1}{2}\left\{\begin{array}{c}
\frac{\partial u}{\partial r} \cdot \frac{\partial u}{\partial r}+\frac{\partial w}{\partial r} \cdot \frac{\partial w}{\partial r} \\
\frac{u}{r} \cdot \frac{u}{r} \\
\frac{\partial u}{\partial z} \cdot \frac{\partial u}{\partial z}+\frac{\partial w}{\partial z} \cdot \frac{\partial w}{\partial z} \\
\frac{\partial u}{\partial r} \cdot \frac{\partial u}{\partial z}+\frac{\partial w}{\partial r} \cdot \frac{\partial w}{\partial z}
\end{array}\right\}
$$

$$
=\frac{1}{2}\left[\begin{array}{ccccc}
\frac{\partial u}{\partial r} & 0 & 0 & \frac{\partial w}{\partial r} & 0 \\
0 & \frac{u}{r} & 0 & 0 & 0 \\
0 & 0 & \frac{\partial u}{\partial z} & 0 & \frac{\partial w}{\partial z} \\
\frac{1}{2} \cdot \frac{\partial u}{\partial z} & 0 & \frac{1}{2} \cdot \frac{\partial u}{\partial r} & \frac{1}{2} \cdot \frac{\partial w}{\partial z} & \frac{1}{2} \cdot \frac{\partial w}{\partial r}
\end{array}\right]\left\{\begin{array}{c}
\frac{\partial u}{\partial r} \\
\frac{u}{r} \\
\frac{\partial u}{\partial z} \\
\frac{\partial w}{\partial r} \\
\frac{\partial w}{\partial z}
\end{array}\right\}
$$


$=\frac{1}{2}[\Delta \theta][G][N]\{\delta\}_{e}$

Where: $[G]=\left[\begin{array}{cc}\frac{\partial}{\partial r} & 0 \\ \frac{1}{r} & 0 \\ \frac{\partial}{\partial z} & 0 \\ 0 & \frac{\partial}{\partial r} \\ 0 & \frac{\partial}{\partial z}\end{array}\right] ;[\Delta \theta]=\left[\begin{array}{ccccc}\frac{\partial u}{\partial r} & 0 & 0 & \frac{\partial w}{\partial r} & 0 \\ 0 & \frac{u}{r} & 0 & 0 & 0 \\ 0 & 0 & \frac{\partial u}{\partial z} & 0 & \frac{\partial w}{\partial z} \\ \frac{1}{2} \cdot \frac{\partial u}{\partial z} & 0 & \frac{1}{2} \cdot \frac{\partial u}{\partial r} & \frac{1}{2} \cdot \frac{\partial w}{\partial z} & \frac{1}{2} \cdot \frac{\partial w}{\partial r}\end{array}\right]$

Therefore, the strain matrix caused by the nonlinear deformation is

$\left[B_{L}\right]_{e}=\frac{1}{2}[\Delta \theta][G][N]$

\section{ACTUAL EXAMPLE ANALYSIS}

\section{Calculating Explanation}

Take the highway engineering around JiNan city for example, the soil is loess with the density of $19.0 \mathrm{kN} / \mathrm{m}^{3}$, the mass of hammer is $98 \mathrm{kN}$ and its cross sectional area is $3.9 \mathrm{~m}^{2}$, the height at which the hammer is dropped is $10 \mathrm{~m}$. The loading modulus is $6000 \mathrm{kPa}$ and the unloading modulus is $24000 \mathrm{kPa}$, the Poisson ration $\mu$ is 0.40 , time step $\Delta t=1 \mathrm{~ms}$. The vertical height of dispersing area of finite element is $\mathrm{H}=16.0 \mathrm{~m}$ and horizontal radius is $5.0 \mathrm{~m}$. The bottom boundary condition of soil is $u=w=0$; the side boundary condition is $w=0$ and contact force of noncompaction area of the surface is zero. Besides, initial conditions are $\left.u\right|_{t=0}=0,\left.\quad w\right|_{t=0}=0,\left.\quad \dot{u}\right|_{t=0}=0,\left.\dot{w}\right|_{t=0}=0$, $\left.\ddot{u}\right|_{t=0}=0$ and $\left.\ddot{w}\right|_{t=0}=0$. The first attack on the compaction is calculated, where the maximum input contact stress is $940 \mathrm{kPa}$ and contact time is $90 \mathrm{~ms}$. Time-interval curves are simplified into three load types, that is, isosceles triangle load, half-sine load with damping and normal distribution curve of load (see Fig. (2). Triangle, sine and normal is called for short below).

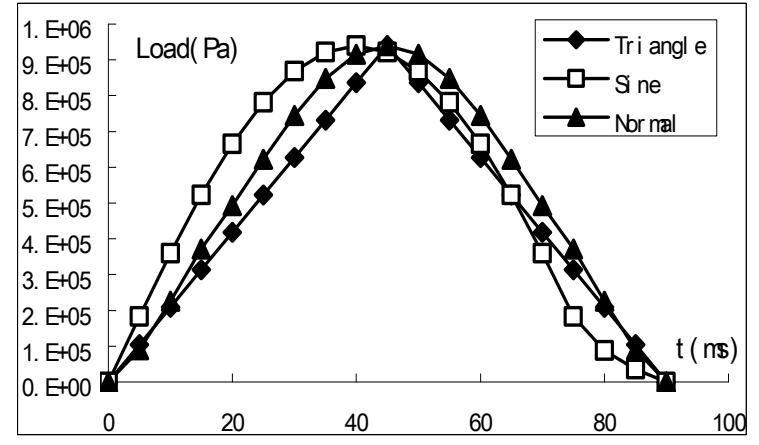

Fig. (2). Time-interval curve of load.

\section{Results and Analyses}

Calculation results on No.265 node (the contact point of Hammer center with the ground surface) are compared and analyzed. Stress and time-interval curves from Fig. (3) to Fig. (5) reflect more consistently that soil has shown a significant rebound at around $60 \mathrm{~ms}$ in the compaction process. Rebound reflected in the displacement curve of Fig. (6) comes later about $10 \mathrm{~ms}$ than that of the stress and timeinterval curve, where rebounds of Triangle, sine and normal occur at $73 \mathrm{~ms}, 70 \mathrm{~ms}$ and $75 \mathrm{~ms}$ respectively.

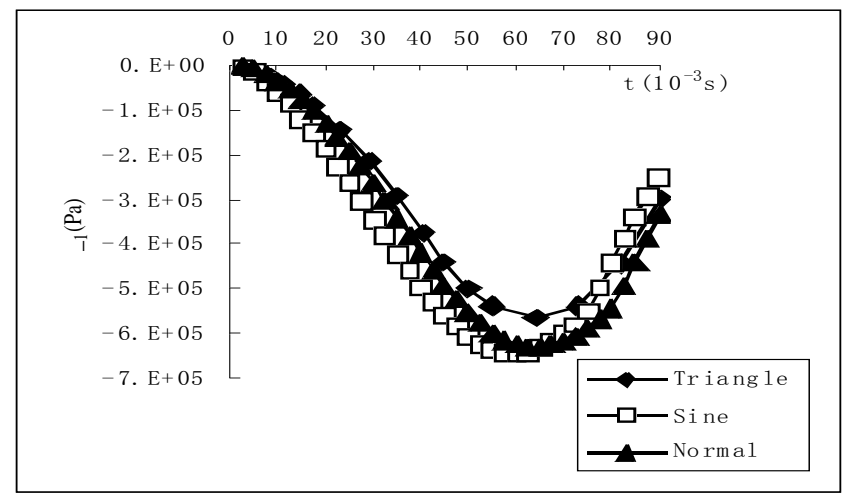

Fig. (3). Time-interval curve of $\sigma_{1}$ for No. 265 node.

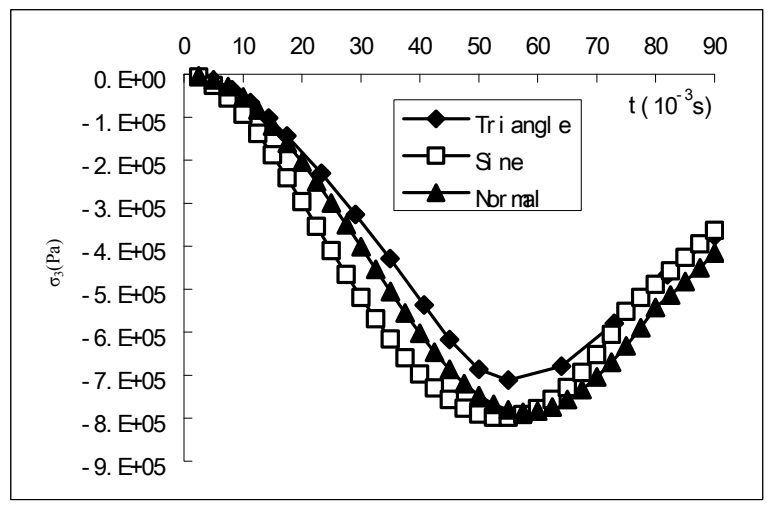

Fig. (4). Time-interval curve of $\sigma_{3}$ for No. 265 node.

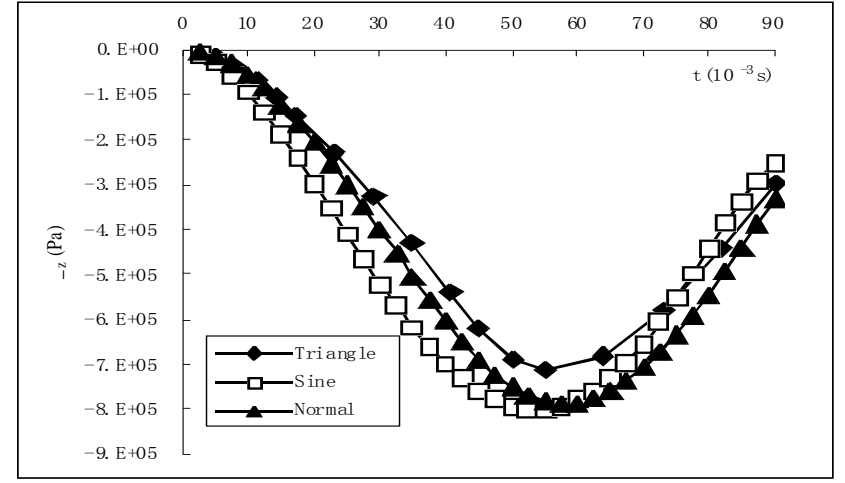

Fig. (5). Time-interval curve of $\sigma_{\mathrm{z}}$ for No. 265 node. 


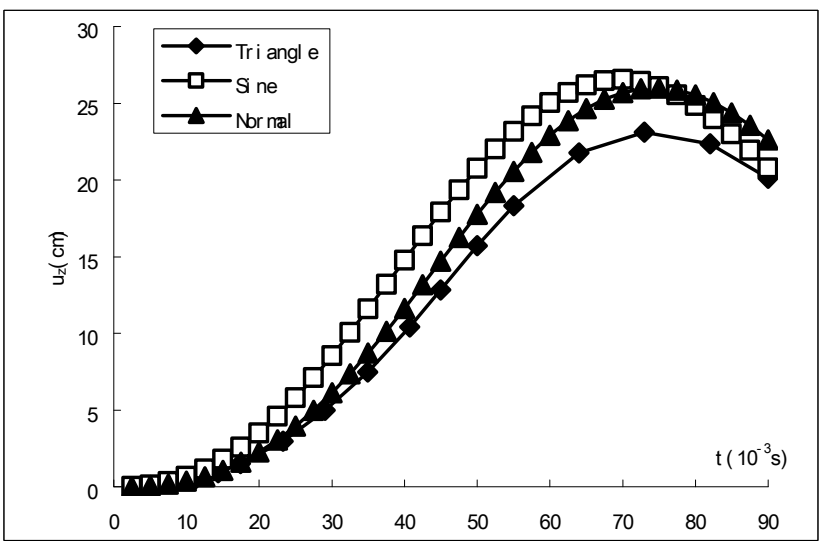

Fig. (6). Time-interval curve of $u_{z}$ for No. 265 node.

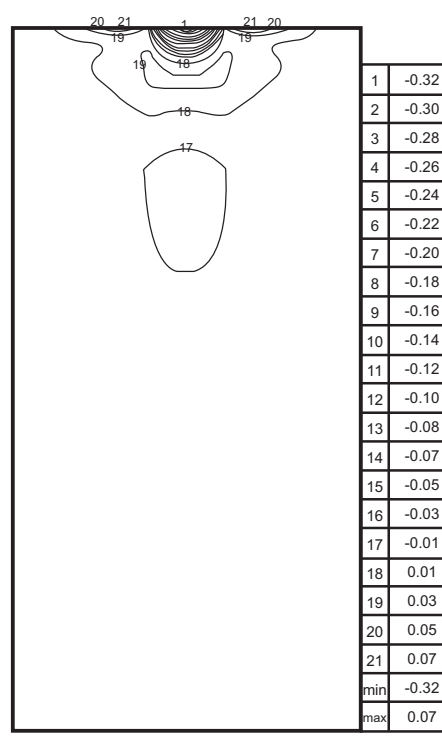

(a) triangle

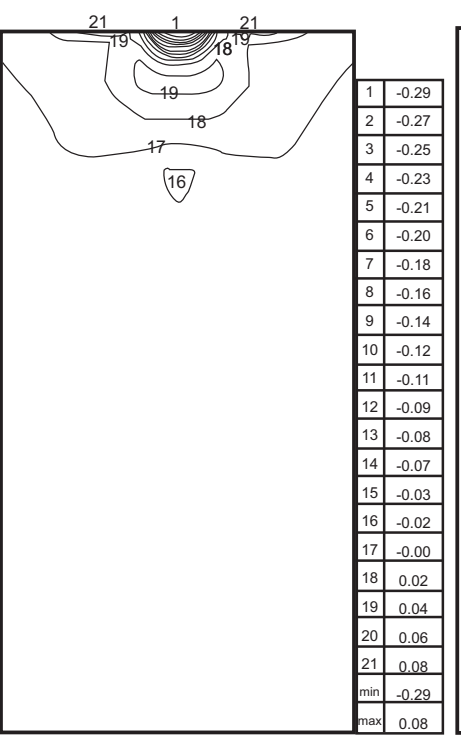

(b) sine

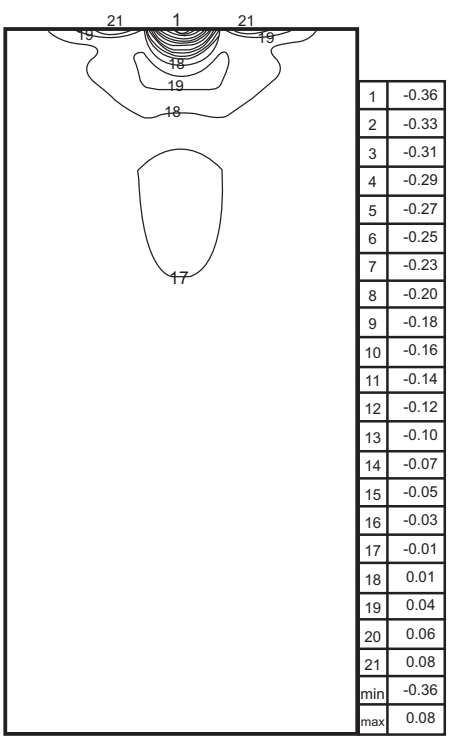

(c) normal

Fig. (7). Contour maps of $\sigma_{1}$ for foundation after compaction (MPa).

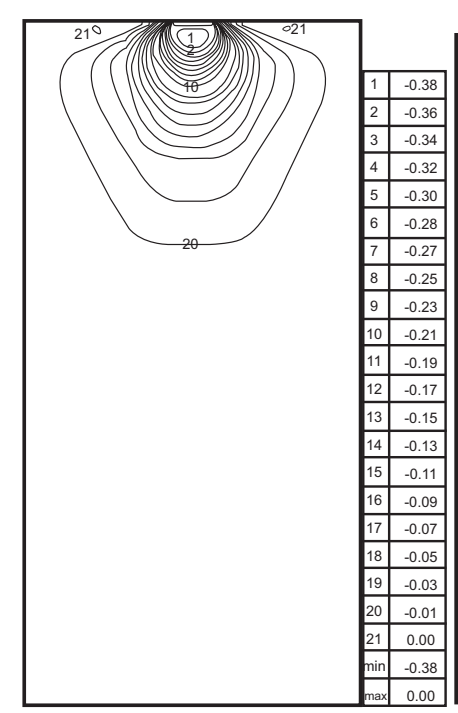

(a) triangle

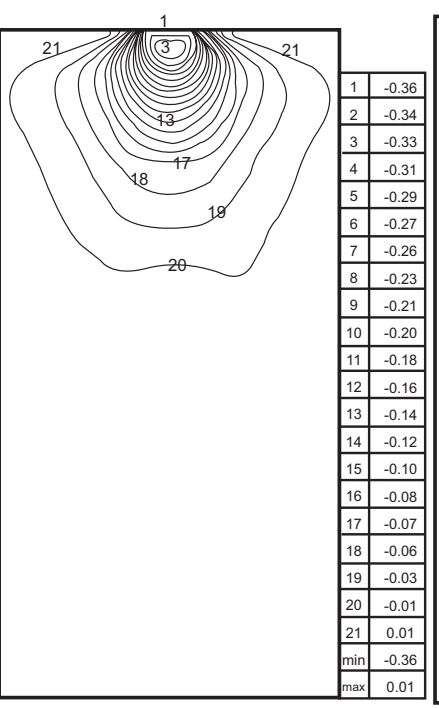

(b) sine

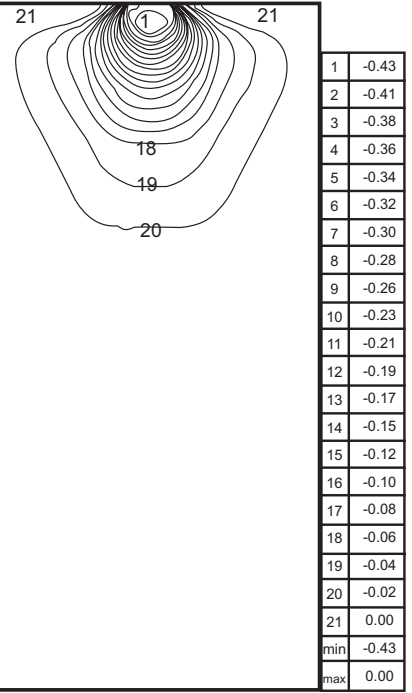

(c) normal

Fig. (8). Contour maps of $\sigma_{3}$ for foundation after compaction (MPa). 
Viewed from the peak of the time-interval curve, the peaks of sine and normal are much closer, where the difference is less than 3\%. Compared them with the triangular peak, the difference is more than $11 \%$. Comparing the degree of rebound under these three cases, we can see that the rebound degree of Sine is relatively large while the rebound degree of Triangle is very close to that of normal. Rebound values of Triangle, sine and normal account for $12.9 \%, 21.7 \%$ and $13.1 \%$ of the peak respectively. According to the stress and time-interval curve and the displacement and time-interval curve, we can obtain that, in the compaction, half sine load with damping is more precise when degree of rebound, rebound time, eventually ramming pit value and the stress-displacement changing process are taken into account for the foundation soil.

Contour maps of $\sigma_{1}, \sigma_{3}$ and $\sigma_{z}$ for foundation after compaction are shown in Figs. (7, 8 and 9). From Figs. (7 and $\mathbf{8}$ ), regularities of distribution of $\sigma_{1}$ and $\sigma_{3}$ for the three cases are consistent, where the value of the normal circumstance is the maximum. From Fig. (9), we can see that distribution rules are basically consistent, where the result of normal distribution curve of load is the maximum and that of sine load is the minimum. From Fig. (10), we can see that

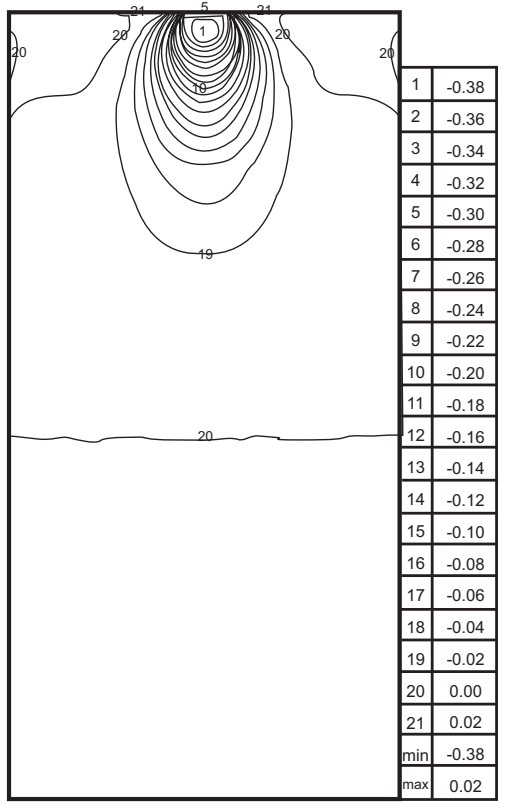

(a) triangle

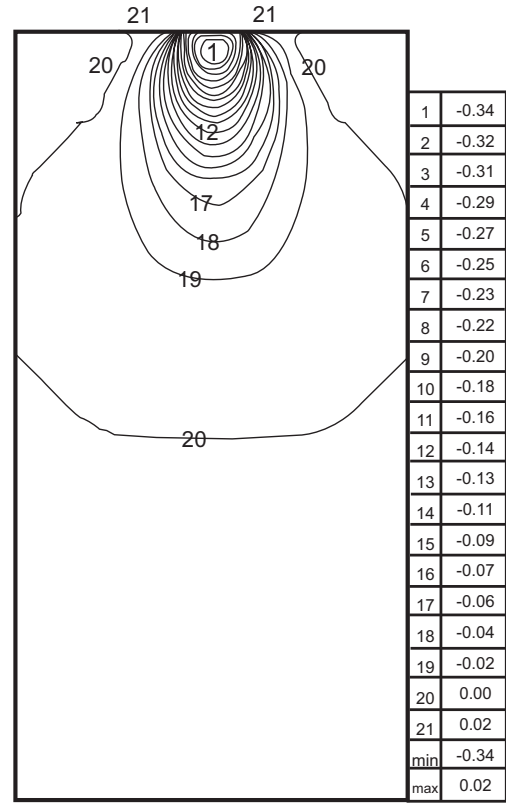

(b) sine

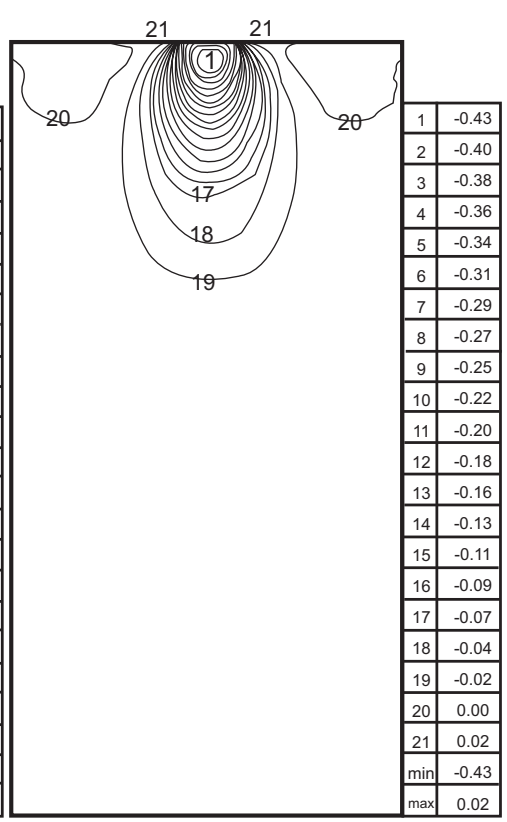

(c) normal

Fig. (9). Contour maps of $\sigma_{z}$ for foundation after compaction (MPa)

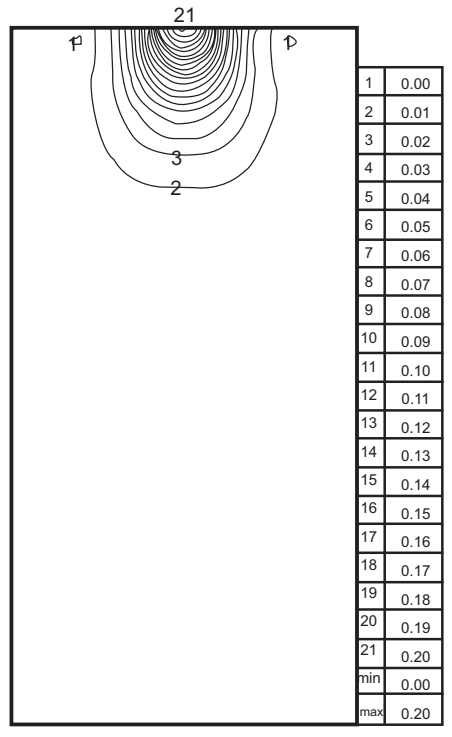

(a) Triangle

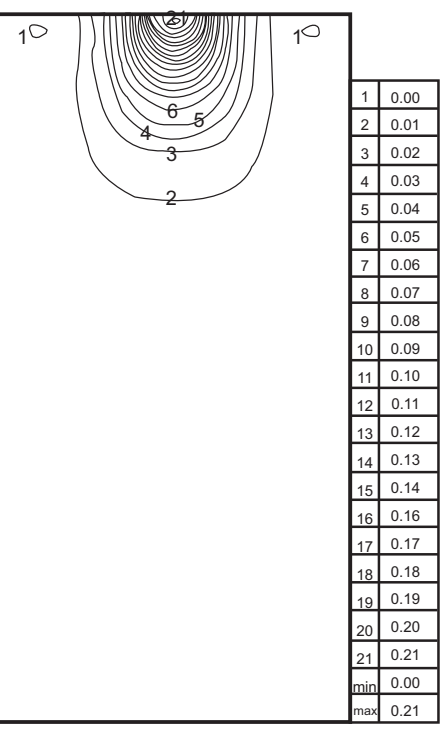

(b) sine

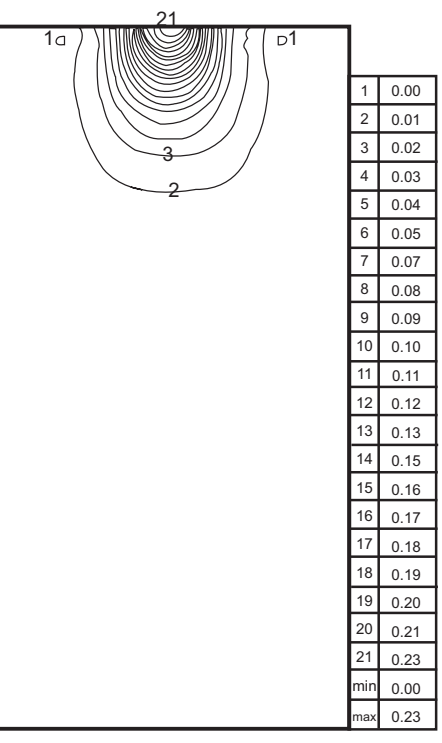

(c) normal

Fig. (10). Contour maps of $u_{z}$ for foundation after compaction (m). 
distribution rules are basically consistent, where the result of normal distribution curve of load is the maximum and that of triangle load is the minimum.

\section{CONCLUSIONS}

In the analysis of dynamic compaction problems, as the geometry non-linearity have been considered, the mechanics analysis is finer, and the model corresponds to the actual feature of dynamic compaction.

Through the analysis and computation, we can get the changing regularity of the soil displacement and dynamic stress on surface during the acting time of dynamic compaction. The effective way of analyzing and simulating dynamic compaction mechanism is raised. The last one should be elucidated is that hypothesis is not considered in dynamic contact condition.

\section{ACKNOWLEDGMENTS}

This paper is supported by the New Century Excellent Talents in University (Grant No.070003), State Key Laboratory of Hydrology-Water Resources and Hydraulic Engineering (Grant No. 2010491011) and the Natural Science Foundation of Anhui Province of China (Grant No.11040606M119).

\section{REFERENCES}

[1] J. H. Qian and F. S. Shuai, "Application of BEM to dynamic consolidation”, Sci. Sin., Vol. 29, pp. 329-336, 1987.

[2] P. Jiang, R. Q. Li and D. F. Kong, "Numerical analysis of large deformation impact and collision properties during dynamic compaction", China J. Geotech. Eng., Vol.22, pp. 222-226, 2000.

[3] D. S. Kim and J. S. Lee, "Propagation and attenuation characteristics of various ground vibrations", Soil Dyn. Earthquake Eng., Vol. 19, pp. 115-126, 2000.
[4] Y. C. Li, J. Chen and S. H. Zhou, "Numerical simulation for a foundation reinforcement procedure using dynamic consolidation technique", Rock. Soil. Mech., Vol. 22, pp. 6-11, 2001.

[5] J. Yang and S. M. Wu, "Transient solutions of axisymmetric dynamic problem for inhomogeneous two phase media", Acta. Mech. Sin., Vol. 28, pp. 308-318, 1996.

[6] L. W. Kong, "An improvement of transient solutions of axisymmetric dynamics problem for two phase media", Acta. Mech. Sin., Vol. 30, pp. 86-92, 1998.

[7] J. Chen, Y. C. Li and S. H. Zhou, "Numerical simulation for foundation reinforcement procedure using dynamic consolidation technique", J. Shanghai. Tiedao. Uni., Vol. 21, pp. 1-7, 2000.

[8] Z. Z. Ding and Y. R. Zhen, "Numerical simulation of saturated soft clay ground improvement by DCM", China J. Under. Spa. Eng., Vol.22, pp. 137-141, 2002.

[9] X. G. Song, Y. Y. Li and J. Han, "Multi-coupling analysis of dynamic consolidation method for reinforcing foundations", Rock. Soil. Mech., Vol. 24, pp. 471-474, 2003.

[10] M. Gunaratne, M. Rangansth, S. Thilakasiri, etal, "Study of pore pressures induced in laboratory dynamic consolidation", Comput. Geotech., Vol. 18, pp. 127-143, 1996.

[11] X. L. Zhou, J. H. Wang and J. F, "Lu Transient dynamic response of poroelastic medium subjected to impulsive loading", Comput. Geotech., Vol. 30, pp. 109-120, 2003.

[12] X. L. Zhou, J. H. Wang and J. F. Lu, "Transient foundation solution of saturated soil to impulsive concentrated loading", Soil Dyn. Earthquake Eng., Vol. 22, pp. 273-281, 2002.

[13] J. L. Pan and A. R. Selby, "Simulation of dynamic compaction of loose granular soils", Adv. Eng. SoftWare., Vol. 33, pp. 631-640, 2002.

[14] R. A. Scott and R. W. Pearce, "Soil compaction by impact", Geo. Technique, Vol. 25, pp. 19-30, 1975.

[15] L. W. Kong and J. X. Yuan , "Study on surface contact stress distribution properties for multi-layered foundation during dynamic consolidation", Acta Mech. Sin., Vol. 31, pp. 250-256, 1999.

[16] J. M. Roesset, "Impact of weight falling onto the ground", $J$. Geotech. Eng., Vol. 120, pp. 1394-1412, 1994.

[17] P. Jiang and R. Q. Li, "Numerical analysis of larger deformation impact and collision properties during dynamic compaction", China J. Geotech. Eng., Vol. 22, pp. 222-226, 2000.

() Nenggang et al.; Licensee Bentham Open.

This is an open access article licensed under the terms of the Creative Commons Attribution Non-Commercial License (http://creativecommons.org/licenses/_by-nc/3.0/) which permits unrestricted, non-commercial use, distribution and reproduction in any medium, provided the work is properly cited. 